\title{
XPC-Related Protection Against Carcinogen-Induced Lung Adenocarcinoma is Independent of Lung Inflammation
}

Isaac Lamb ${ }^{1}$, Huaxin Zhou ${ }^{2}$, Patricia Smith², and Catherine R. Sears ${ }^{2}$, M.D.

${ }^{1}$ Indiana University School of Medicine, ${ }^{2}$ Indiana University School of Medicine, Dept. of Medicine,

Division of Pulmonary, Critical Care, Sleep and Occupational Medicine

Background and Hypothesis: Cigarette smoke (CS) exposure causes lung cancer, with both DNA damage and local inflammation playing a critical role in development. Our previous research links the DNA repair protein Xeroderma Pigmentosum Group C (XPC) with protection against lung cancer in CS- and carcinogen-exposed mouse models. In mice (XPC-deficient and wild-type [WT] littermates) exposed to continuous CS for 9 months, neither XPC-deficient nor WT mice develop lung cancer. XPC-deficient but not WT mice exposed to 5 months CS +4 months air control (AC) (recovery model) develop lung cancer. In a direct carcinogen model, XPC-deficiency accelerates NTCU lung squamous cell development. Our hypothesis is that lung cancers in XPC-deficient mice are independent of treatment alterations in BAL inflammation.

Experimental Design or Project Methods: Acellular bronchoalveolar lavage (BAL) samples from the three different mouse CS and carcinogen models were analyzed for the inflammatory cytokines IL-6, IL10, IL-12p70, IL-17A, IFN- $\gamma$, MCP-1, and TNF- $\alpha$ by a cytometric bead array (BD Biosciences) using a flow cytometer (FACScan) according to the manufacturer's instructions. Raw data (mean fluorescence intensity) was analyzed by BD CBA Software. Statistical comparisons were by ANOVA, with $p<0.05$ considered significant.

Results: All measured cytokine levels were low or undetectable in BAL from AC and CS mice, with no significant XPC genotype or treatment-related changes in the measured cytokines. Differences were observed in TNF- $\alpha$ and IL- 6 between continuous and recovery CS models independent of treatment or genotype. NTCU caused a significant increase in BAL IL-6 independent of genotype. IL-17a was elevated in NTCU-treated mice that developed lung squamous cell carcinoma.

Conclusion and Potential Impact: The XPC genotypic variation seen in lung carcinogenesis appears to be independent of BAL cytokines, suggesting that the variation is due to DNA damage rather than differences in local inflammation. Further mechanistic investigations will focus on DNA damage and repair as drivers of XPC-deficient lung cancers. 